\title{
ON GENERATING REGULAR CANTORVALS CONNECTED WITH GEOMETRIC CANTOR SETS
}

\author{
ARTUR BARTOSZEWICZ, MAŁGORZATA FILIPCZAK, SZYMON GŁA̧B, \\ FRANCISZEK PRUS-WIŚNIOWSKI, AND JAROSŁAW SWACZYNA
}

\begin{abstract}
We show that the Cantorvals connected with the geometric Cantor sets are not achievement sets of any series. However many of them are attractors of IFS consisting of affine functions.
\end{abstract}

\section{INTRODUCTION}

Let $\left(x_{n}\right)=\left(x_{1}, x_{2}, \ldots\right)$ be an absolutely summable sequence, in symbols $\left(x_{n}\right) \in \ell_{1}$, and let

$$
E\left(x_{n}\right)=\left\{\sum_{n=1}^{\infty} \varepsilon_{n} x_{n}:\left(\varepsilon_{n}\right) \in\{0,1\}^{\mathbb{N}}\right\}
$$

denote the set of all subsums of the series $\sum_{n=1}^{\infty} x_{n}$. The set $E\left(x_{n}\right)$ is called the achievement set of $\left(x_{n}\right)$ (see [12]). It is easy to see that for $\left(x_{n}\right)=\left(\frac{2}{3}, \frac{2}{3^{2}}, \ldots\right)$ the set $E\left(x_{n}\right)$ is equal to the classic Cantor ternary set $C$ and for $\left(x_{n}\right)=\left(\frac{1}{2}, \frac{1}{2^{2}}, \ldots\right)$ we have $E\left(x_{n}\right)=[0,1]$. Achievement sets of sequences have been considered by many authors, some results have been rediscovered several times. The following properties of sets $E\left(x_{n}\right)$ were described by Kakeya in [13] in 1914:

(i) $E\left(x_{n}\right)$ is a compact perfect set,

(ii) If $\left|x_{n}\right|>\sum_{i>n}\left|x_{i}\right|$ for all sufficiently large $n$ 's, then $E\left(x_{n}\right)$ is homeomorphic to the Cantor set $C$,

(iii) If $\left|x_{n}\right| \leq \sum_{i>n}\left|x_{i}\right|$ for all sufficiently large $n$ 's, then $E\left(x_{n}\right)$ is a finite union of closed intervals. Moreover, if $\left|x_{n}\right| \geq\left|x_{n+1}\right|$ for all but finitely many $n$ 's and $E\left(x_{n}\right)$ is a finite union of closed intervals, then $\left|x_{n}\right| \leq \sum_{i>n}\left|x_{i}\right|$ for all but finitely many $n$ 's.

One can see that $E\left(x_{n}\right)$ is finite if and only if $x_{n}=0$ for all but finite number of $n$ 's, i.e. $\left(x_{n}\right) \in c_{00}$. Kakeya conjectured that if $\left(x_{n}\right) \in \ell_{1} \backslash c_{00}$, then $E\left(a_{n}\right)$ is always homeomorphic to the Cantor set $C$ or it is a finite union of intervals. In 1980 Weinstein and Shapiro in [18] gave an example whose showed that the Kakeya hypothesis is false. Independently, the similar example was given by Ferens in [8]. In [9] Guthrie and Nymann gave a very simple example of a sequence whose achievement set is not a finite union of closed intervals but it has nonempty interior. They used the following sequence $\left(t_{n}\right)=\left(\frac{3}{4}, \frac{2}{4}, \frac{3}{16}, \frac{2}{16}, \ldots\right)$. Moreover, they formulated the following:

Theorem 1. For any $\left(x_{n}\right) \in \ell_{1} \backslash c_{00}$, the set $E\left(x_{n}\right)$ is one of the following types:

2010 Mathematics Subject Classification. 40A05, 11B05, 28A75, 28A80.

Key words and phrases. Cantorval, achievement set, attractor of IFS.

The last author has been supported by the NCN Grant ("Diamond grant") No. 0168/DIA/2014/43. 
(i) a finite union of closed intervals,

(ii) homeomorphic to the Cantor set $C$,

(iii) homeomorphic to the set $\mathbf{T}=E\left(t_{n}\right)=E\left(\frac{3}{4}, \frac{2}{4}, \frac{3}{16}, \frac{2}{16}, \frac{3}{64}, \ldots\right)$.

Although their proof had a gap, the theorem is true and the correct proof was given by Nymann and Saenz in [17]. Guthrie, Nymann and Saenz have observed that the set $\mathbf{T}$ is homeomorphic to the set $N$ described by the formula

$$
N=[0,1] \backslash \bigcup_{n \in \mathbb{N}} U_{2 n}
$$

where $U_{n}$ denotes the union of $2^{n-1}$ open middle thirds which are removed from the interval $[0,1]$ at the $n$-th step in the construction of the classic Cantor ternary set $C$. Such sets are called Cantorvals in the literature (to emphasize the similarity to the interval and to the Cantor set simultaneously). It is known that a Cantorval is such nonempty compact set in $\mathbb{R}$, that it is the closure of its interior and both endpoints of any nontrivial component are accumulation points of its trivial components. Other topological characterizations of Cantorvals can be found in [5] and [14]. Let us observe that Theorem 1 states that $\ell_{1}$ can be divided into four disjoint sets $c_{00}, \mathcal{C}, \mathcal{J}$ and $\mathcal{M C}$, where $\mathcal{J}$ consists of sequences with achievement sets being finite unions of intervals, $\mathcal{C}$ consists of sequences $\left(x_{n}\right)$ with $E\left(x_{n}\right)$ homeomorphic to the Cantor set and $\mathcal{M C}$ consists of the sequences with achievement sets homeomorphic to the set $\mathbf{T}$ or equivalently to $N(\mathcal{M C}$ is the abbrevation of Middle-Cantorval because the structure of the achievement set should be symmetric). Algebraic and topological properties of these subsets of $\ell_{1}$ have been recently studied in [2]. All known examples of sequences which achievement sets are Cantorvals belong to the class of multigeometric sequences. This class was deeply investigated in [12], [6] and [1]. In particular, the achievement sets of multigeometric series and sets obtained in more general case are the attractors of the affine iterated function systems, see [1]. More information on achievement sets can be found in the surveys [5], [15] and [16]. The aim of our paper is to show that the most known Cantorval $N=[0,1] \backslash \bigcup_{n \in \mathbb{N}} U_{2 n}=C \cup \bigcup_{n \in \mathbb{N}} U_{2 n-1}$, and the other members of large class of Cantorvals connected with geometric Cantor sets are not isometric to an achievement set for any sequence (section 2) but they are attractors of iterated function system (IFS) consisting of several affine functions (section 3).

It is almost obvious that any achievement set $E$ of a summable sequence contains zero and is symmetric in the sense that there exists a number $t$ such that if $t-x \in E$ then $t+x \in E$ too. It is a natural question if every set with these properties is an achievement set for some sequence. This question was posted by W. Kubiś in Łódź in 2015.In particular, in [7] the authors ask if the Cantorval $N$ is an achievement set of any sequence.

Recently, the authors of [7] have considered the N-G-Cantorval $\mathbf{T}$ (defined in Theorem 1 (iii)), the Cantor set $Y=\partial \mathbf{T}$ and the Cantorval $Z$ whose intervals are the gaps of $\mathbf{T}$, and the gaps of $Z$ are the intervals of $\mathbf{T}$. They introduced the notion of center of distances and used it to prove that $Y$ and $Z$ are not achievement sets for any series. Recall that the center of distances of a metric space $(X, d)$ is the set

$$
C(X)=\{\alpha \geq 0: \forall x \in X \exists y \in X, \text { such that } d(x, y)=\alpha\} .
$$


Clearly for $X=E\left(x_{n}\right)$ for a sequence $\left(x_{n}\right)$ of positive terms we have $\left\{x_{n}: n \in \mathbb{N}\right\} \subset C(X)$. If we assume that $N=E\left(x_{n}\right)$, we conclude by the Second Gap Lemma (Lemma 4 in the second section) that $x_{n}=\frac{2}{9}$ for some $n \in \mathbb{N}$. But for $x=\frac{25}{54}$ we observe that $x \in N$ and the set $\left\{y \in N:|x-y|=\frac{2}{9}\right\}=\emptyset$. Therefore $N$ is not an achievement set. We are not able to use this method to prove our main theorem but the result itself has a similar nature. Our result and that from [7] suggest the following:

Conjecture 2. Let $X$ be a Cantor set and $Y, Z$ be Cantorvals with $X=\partial Y=\partial Z$. Then at most one set of $X, Y, Z$ can be an achievement set.

\section{MAin Results}

Let us assume that $\left(x_{n}\right)$ is a nonincreasing, absolutely summable sequence of positive real numbers. Denote (as in [9], [17], [5]):

$$
\begin{aligned}
& E=E\left(x_{n}\right)=\left\{\sum_{n=1}^{\infty} \varepsilon_{n} x_{n}:\left(\varepsilon_{n}\right) \in\{0,1\}^{\mathbb{N}}\right\} ; \\
& E_{k}=\left\{\sum_{n=k+1}^{\infty} \varepsilon_{n} x_{n}\right\} ; F_{k}=\left\{\sum_{n=1}^{k} \varepsilon_{n} x_{n}\right\} .
\end{aligned}
$$

Of course we have

$$
\begin{aligned}
& E=E_{k}+F_{k}=\bigcup_{f \in F_{k}}\left(f+E_{k}\right) \\
& E_{k}=E_{k+1} \cup\left(x_{k+1}+E_{k+1}\right)
\end{aligned}
$$

for all $k \in \mathbb{N}$. Moreover, let $r_{k}:=\sum_{n=k+1}^{\infty} x_{n}$. By a gap in $E$ we understand any such interval $(a, b)$ that $a \in E, b \in E$ and $(a, b) \cap E=\emptyset$. The following two lemmas can be found in [5]. We provide their proofs for reader's convenience. The first is obvious.

Lemma 3. (First Gap Lemma) If $x_{k}>r_{k}$ then $\left(r_{k}, x_{k}\right)$ is a gap in E.

The next observation is extracted from the proof of the crucial Lemma 4 of [17], where it was formulated as not a quite correct claim (however the Lemma and the main result of [17] are true).

Lemma 4. (Second Gap Lemma) Let $(a, b)$ be a gap in E, and let $p$ be defined by the formula $p:=\max \left\{n: x_{n} \geq b-a\right\}$. Then:

(i) $b \in F_{p}$,

(ii) If $F_{p}=\left\{f_{1}^{(p)}<f_{2}^{(p)}<\ldots<f_{m(p)}^{(p)}\right\}$ and $b=f_{j}^{(p)}$, then $a=f_{j-1}^{(p)}+r_{p}$.

Proof. Let us observe that $b \in F_{p}$. If not, we have $b=\sum_{n \in A} x_{n}$, where $A$ contains some $i$ greater than $p$. Hence $x_{i}<b-a$ and $b-x_{i} \in E$. Consequently we obtain the element $b-x_{i} \in E$ which belongs to the gap $(a, b)$, contradiction. So let $b=f_{j}^{(p)}$. Then, by the definition of a gap, we have $a \geq f_{j-1}^{(p)}$. Now we will show that $f_{j-1}^{(p)}+r_{p} \leq b$. Suppose $f_{j-1}^{(p)}+r_{p}>b$. Let us consider the sequence $\left(f_{j-1}^{(p)}+r_{i}\right)_{i=p}^{\infty}$ which is decreasing and converging to $f_{j-1}^{(p)}$. Since the difference between the consecutive terms of this sequence is smaller than $b-a$ (by the definition 
of $p$ ), there exists a term which belongs to $(a, b)$, which gives a contradiction. Consequently $f_{j-1}^{(p)}+r_{p} \leq a$. On the other hand $a<f_{i}^{(p)}$ for $i \geq j$ and hence $a \in \bigcup_{i<j}\left(f_{i}^{(p)}+E_{p}\right)$. Therefore $a \leq \sup \left(f_{j-1}^{(p)}+E_{p}\right)=f_{j-1}^{(p)}+r_{p}$.

For our purpose we need the following strenghtening of the Second Gap Lemma.

Lemma 5. Suppose that $(a, b)$ is a gap in $E=E\left(x_{n}\right)$ such that for any gap $\left(a_{1}, b_{1}\right)$ with $b_{1}<a$ we have $b-a>b_{1}-a_{1}$ (in other words $(a, b)$ is the longest gap from the left). Then $b=x_{k}$ for some $k \in \mathbb{N}$ and $a=r_{k}$.

Proof. By the Second Gap Lemma $b$ is a finite sum of terms of $\left(x_{n}\right)$. Let $b=x_{n_{1}}+\ldots+x_{n_{m}}$ with $x_{n_{1}} \geq \ldots \geq x_{n_{m}}$. Suppose that $m \geq 2$. Firstly observe that $x_{n_{m}} \geq b-a$ (indeed, if $x_{n_{m}}<b-a$ then $b-x_{n_{m}} \in(a, b) \cap E$ which is impossible). Of course $x_{n_{m}}<b$ and, since $(a, b)$ is a gap, $x_{n_{m}} \leq a$. Any gap in the set $X:=E \cap\left[0, x_{n_{m}}\right]$ is shorter than $b-a$. On the other hand, $b \in X+\left(b-x_{n_{m}}\right)$ and $X+\left(b-x_{n_{m}}\right) \subset E$, so $(a, b) \cap\left(X+\left(b-x_{n_{m}}\right)\right)=\emptyset$, a contradiction. Thus $m=1$ which means that $b=x_{k}$ for some $k \in \mathbb{N}$.

Since $a \in E, r_{k} \geq a$. Suppose that $r_{k}>a$. Let $m$ be the smallest number satisfying $\sum_{n=k+1}^{m} x_{n}>a$. Hence $\sum_{n=k+1}^{m} x_{n}>b$, because $(a, b)$ is a gap. Let now $X:=E \cap\left[0, x_{m}\right]$. Then the set $X+\sum_{n=k+1}^{m-1} x_{n}$ is included in $E$ and has allgaps shorter than $b-a$, which gives a contradiction again.

Note that for $q \geq 1 / 2$ the achievement set $E\left(q^{n}\right)$ is an interval. For $q \in(0,1 / 2)$ let $C_{q}=E\left((1-q) q^{n-1}\right)$. Then $C_{q}=[0,1] \backslash \bigcup_{n=1}^{\infty} U_{n}^{q}$ where $U_{n}^{q}$ is a union of $2^{n-1}$ open intervals, each of the length $q^{n-1}(1-2 q)$, removed from $[0,1]$ in the $n$-th step in $C_{q}$ construction, exactly as in the construction of the classic Cantor set $C=C_{1 / 3}$.

Now, let us divide $\mathbb{N}$ into two infinite sets: $H$ and its complement $H^{c}$. Consider the set $N_{q}^{H}=[0,1] \backslash \bigcup_{n \in H} U_{n}^{q} . N_{q}^{H}$ is evidently a Cantorval and it is regular in the sense that in each step of the construction we remove or we leave all the components of the set $U_{n}^{q}$. Let us observe that the Cantor set $C_{q}$ is the boundary of any Cantorval $N_{q}^{H}$. For $H=2 \mathbb{N}$ we write simply $N_{q}$ instead of $N_{q}^{2 \mathbb{N}}$. Note that $N=N_{1 / 3}$ is the generic example of Cantorval considered by Guthrie and Nymann which was proved to be homeomorphic with $\mathbf{T}=E\left(\frac{3}{4}, \frac{2}{4}, \frac{3}{16}, \frac{2}{16}, \frac{3}{64}, \ldots\right)$. Thus the sets $N_{q}^{H}$ form a wide class of Cantorvals containing the very important example. In the following figure is presented the nested construction of the set $N_{q}$, for some $q \leq 1 / 3$.

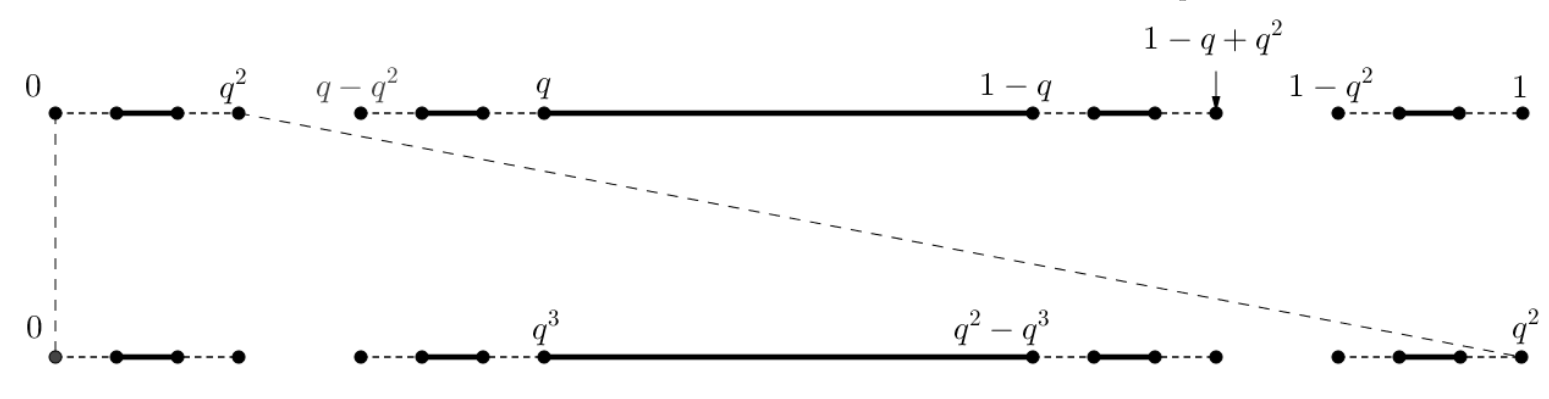

Figure 1

In the next lemma we will consider the longest interval which is contained in the finite union of translations of $N_{q}^{H}$. It is easy to see that if $1 \notin H, J=[q, 1-q]$ and $t=1-2 q$, then the 
longest interval $I$ which is contained in $N_{q}^{H} \cup\left(t+N_{q}^{H}\right)$ might have length $2|J|$. However, it can be a bit longer. For example, if $\left(t+N_{q}^{H}\right)$ contains the component $[a, b]$ with $a<q \leq b$ then $\inf I<\inf J$. In the analogous way $\sup I$ might be bigger than $\sup (t+J)$. What is even more interesting, there are such $q$ 's for which $\sup J<\inf (t+J)$ and $J \cup(t+J) \subset I$ - see Figure 2 .

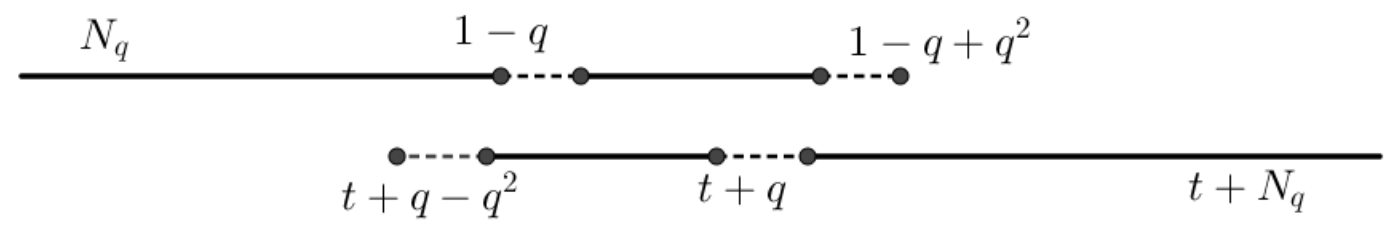

Figure 2

Such a situation can be observed, for example, for $q=1 / 4$. One can see that $N_{\frac{1}{4}} \cup\left(N_{\frac{1}{4}}+\frac{35}{64}\right)$ contains an interval $I$ with $|I|>2|J|$. In fact $|I|=2 \cdot \frac{1}{2}+\frac{3}{64}$.

Lemma 6. Let $N_{q}^{H}$ be a regular Cantorval, $1 \notin H, 2 \in H$.

(i) If $q \leq 1 / 3$ and $0=t_{1}<t_{2}<\cdots<t_{l}$ for some $l \in \mathbb{N}$, then the length of the longest interval contained in the union of translations $\bigcup_{i=1}^{l}\left(t_{i}+N_{q}^{H}\right)$ is smaller than $\left(l(1-2 q)+(l-1) q^{2}\right)$.

(ii) If $q \in(1 / 3, \sqrt{2}-1)$ and $t>0$ then the length of the longest interval contained in $N_{q}^{H} \cup$ $\left(t+N_{q}^{H}\right)$ is smaller than $5(1-2 q)$.

(iii) If $q \in(\sqrt{2}-1,1 / 2)$ and $t>0$ then the length of the longest interval contained in $N_{q}^{H} \cup$ $\left(t+N_{q}^{H}\right)$ is smaller than $4(1-2 q)$.

Proof. Denote by $J$ the longest sub-interval $[q, 1-q]$ of $N_{q}^{H}$ and by $I$ the longest interval contained in $N_{q}^{H} \cup\left(t+N_{q}^{H}\right)$.

(i) First, fix $t>0$ and consider the union $N_{q}^{H} \cup\left(t+N_{q}^{H}\right)$. If $t=1-2 q=|J|$ then $|I|=2|J|$. If $t<|J|$ then there may exist intervals $K_{1} \subset t+N_{q}^{H}$ and $K_{2} \subset N_{q}^{H}$ such that $q \in K_{1}$ and $\inf K_{1}<q$, and $t+1-q \in K_{2}$ and $\sup K_{2}<t+1-q$. Since $\left|K_{1}\right| \leq q^{3}$ and $\left|K_{2}\right| \leq q^{3}$, we obtain

$$
|I|<2|J|+2 q^{3}<2|J|+q^{2} .
$$

Suppose now that $t>|J|$ and remind that $\left(1-q-q^{2}, 1-q^{2}\right)$ is a gap in $N_{q}^{H}$. It follows that if $t-|J|>q^{2}$ then $\left[1-q+q^{2}, t+q\right] \backslash\left(N_{q}^{H} \cup\left(t+N_{q}^{H}\right)\right) \neq \emptyset$. It means that $|I|<2|J|+q^{2}$.

Let $0=t_{1}<t_{2}<t_{3}$ and denote by $I$ the largest interval contained in the $\bigcup_{i=1}^{3}\left(t_{i}+N_{q}^{H}\right)$ (if there is more then one such interval, we denote by $I$ the right one). Denote by $d$ the number $3(1-2 q)+2 q^{2}$. If $t_{2}<|J|+q^{2}$ and $t_{3}<t_{2}+|J|+q^{2}$ then (in the same manner as in the previous consideration) we obtain that $|I|<d$. If $t_{3}>t_{2}+|J|+q^{2}$ then $I=\left(t_{3}+J\right)$ or $I \subset N_{q}^{H} \cup\left(t_{2}+N_{q}^{H}\right)$. In both cases $|I|<d$. Finally, if $t_{2}>|J|+q^{2}$ then $J \cap I=\emptyset$ or the set $t_{3}+N_{q}^{H}$ "fills the gap" between $J$ and $t_{2}+J$, which is possible only if $t_{3}<(1-q)+q^{2}$. In any case $|I|<d$.

In a similar way one can check that for any $l \in N$ and $0<t_{1}<t_{2}<\cdots<t_{l}$ the length of the largest interval contained in the union of translations $\bigcup_{i=1}^{l}\left(t_{i}+N_{q}^{H}\right)$ is not greater than $l(1-2 q)+(l-1) q^{2}$. 
(ii) Note that for any $q \in(0,1 / 2)$ the length of $I$ is smaller then $2|J|+3 q^{2}$. Moreover, for $q<\sqrt{2}-1$, the interval $J$ is longer then $q^{2}$. Therefore, for such $q$ 's, $|I|<5|J|$.

(iii) Suppose now that $q \geq \sqrt{2}-1>2 / 5$. Consequently, $|J|=1-2 q<1 / 5$ and for any component $K$ of $N_{q}^{H}$, the distance between $K$ and a component longer than $K$ is bigger than $2|K|$.

Case 1. $t \leq|J|$. We will show that $\sup I<(2-3 q)+|J|$. If there is no component $[a, b]$ of $N_{q}^{H}$ such that $a \leq \sup (t+J)<b$, then $\sup I=\sup (t+J) \leq 2-3 q$. If such a component exists, denote it by $\left[a_{1}, b_{1}\right]$. Note that $b_{1}-a_{1} \leq q^{2}|J|$, since the longest component of $N_{q}^{H} \cap[1-q, 1]$ is not bigger than $q^{2}|J|$. If there exists a component $\left[a_{2}, b_{2}\right]$ of $t+N_{q}^{H}$ such that $a_{2} \leq b_{1}<b_{2}$ then $b_{2}-a_{2} \leq q\left(b_{1}-a_{1}\right)$. Inductively, if there exists a component $\left[a_{n+1}, b_{n+1}\right]$ such that $a_{n+1} \leq b_{n}<b_{n+1}$, then $b_{n+1}-a_{n+1}<q\left(b_{n}-a_{n}\right)$. If the sequence $\left(\left[a_{n}, b_{n}\right]\right)$ is finite and $\left[a_{n_{0}}, b_{n_{0}}\right]$ is its last element, then $\sup I=b_{n_{0}}<(2-3 q)+q|J|$. If not, we have to look more closely at the structure of $N_{q}^{H}$.

Since the set $H$ is infinite, there is a gap $(\alpha, \beta)$ in $N_{q}^{H} \cap\left[0, \frac{1}{2}|J|\right]$. Moreover, there is $n_{0}$ such that $\left|K_{n}\right|<\beta-\alpha$ for any $n>n_{0}$. Hence

$$
\sup I<b_{n_{0}}+\frac{1}{2}|J|<(2-3 q)+|J|
$$

In the same manner we check that $\inf I>\inf J-|J|$. Finally $\sup I-\inf I<2-3 q+|J|-$ $(q-|J|)=2-4 q+2|J|=4|J|$.

Case 2. $t>|J|$. In this case, using analogous arguments as in Case 1 one can prove that $[1-q, t+q] \backslash\left(N_{q}^{H} \cup\left(t+N_{q}^{H}\right)\right) \neq \emptyset$. Indeed, suppose that $1-q<t+q$. If there is no component $[a, b]$ of $t+N_{q}^{H}$ such that $a \leq 1-q<b$, then $[1-q, t+q] \backslash\left(N_{q}^{H} \cup\left(t+N_{q}^{H}\right)\right) \neq \emptyset$. If such a component exists, denote it by $\left[a_{1}, b_{1}\right]$. Note that $b_{1}-a_{1}<\frac{1}{2}\left(t+q-b_{1}\right)$. If there exists a component $\left[a_{2}, b_{2}\right]$ of $N_{q}^{H}$ such that $a_{2} \leq b_{1}<b_{2}$ then $b_{2}-a_{2}<q\left(b_{1}-a_{1}\right)$. Inductively, if there exists a component $\left[a_{n+1}, b_{n+1}\right]$ such that $a_{n+1} \leq b_{n}<b_{n+1}$, then $b_{n+1}-a_{n+1}<$ $q\left(b_{n}-a_{n}\right)$. Moreover, there is a gap in $N_{q}^{H} \cap[0, t+q-(q-1)]$. In consequence, there is a gap in $[1-q, t+q] \cap\left(N_{q}^{H} \cup\left(t+N_{q}^{H}\right)\right)$. It means that $J$ and $t+J$ are contained in different components of $N_{q}^{H}$. Therefore $|I|<3|J|$ which ends the proof.

Theorem 7. No regular Cantorval $N_{q}^{H}$, with $1 \notin H$ and $2 \in H$, is an achievement set of a sequence.

Proof. Suppose that $N_{q}^{H}=E\left(x_{i}\right)$ for some non-increasing sequence $\left(x_{i}\right)$ with positive terms. Then, by Lemma 5, we conclude that there is $k \in \mathbb{N}$ such that $x_{k}=q-q^{2}$ and $q^{2}=r_{k}$. Since $1=\sum_{j=1}^{\infty} x_{j}$, we have $1-q^{2}=1-r_{k}=x_{1}+x_{2}+\ldots+x_{k} \in F_{k}$. Consequently, $1-q=1-q^{2}-\left(q-q^{2}\right)=x_{1}+x_{2}+\ldots+x_{k-1} \in F_{k-1}$. Hence $F_{k} \cap\left[1-q, 1-q^{2}\right)=\{1-q\}$ and $\left(1-q-\left(q-q^{2}\right), 1-q\right) \cap F_{k-1}=\left((1-q)^{2}, 1-q\right) \cap F_{k-1}=\emptyset$. Since the whole Cantorval $N_{q}^{H}=$ $F_{k}+E_{k}$, the interval $J$ is covered by some number of the sets $\sum_{i=1}^{k} \varepsilon_{i} x_{i}+E_{k} \subset \sum_{i=1}^{k} \varepsilon_{i} x_{i}+\left[0, q^{2}\right]$ for $\left(\varepsilon_{i}\right) \in\{0,1\}^{k}$.

Let $m:=\left|F_{k} \cap\left(q-q^{2}, 2\left(q-q^{2}\right)\right]\right|$. Enumerate $F_{k} \cap\left(q-q^{2}, 2\left(q-q^{2}\right)\right]$ as $\left\{y_{1}<y_{2}<\cdots<y_{m}\right\}$. Note that $x_{k}+x_{k-1} \geq 2\left(q-q^{2}\right)$. Therefore $y_{1}, y_{2}, \ldots, y_{m-1}$ are among elements of the set 
$\left\{x_{i}: x_{i}>x_{k}\right\}$. The element $y_{m}$ can be in $\left\{x_{i}: x_{i}>x_{k}\right\}$ or it can be equal to $x_{k}+x_{k-1}=2\left(q-q^{2}\right)$ if $x_{k}=x_{k-1}$. Now we consider three cases.

Case 1. $m>\frac{1}{q}$ and $\sum_{i=1}^{m} y_{i}>1-q+\left(2 q-2 q^{2}\right)$. Then $\sum_{i=1}^{m-1} y_{i}>1-q$. Note that $\sum_{i=1}^{m-1} y_{i} \in F_{k-1}$. If $\sum_{i=1}^{m-1} y_{i} \in\left(1-q, 1-q^{2}\right)$, we have a contradiction. So suppose that $\sum_{i=1}^{m-1} y_{i} \geq 1-q^{2}$. Let $A \subset\{1,2, \ldots, m-1\}$ be such that $\sum_{i \in A} y_{i} \geq 1-q^{2}$ and for any $B \subset\{1,2, \ldots, m-1\}$ such that $\sum_{i \in B} y_{i} \geq 1-q^{2}$ we have $\sum_{i \in B} y_{i} \geq \sum_{i \in A} y_{i}$. Clearly $|A| \geq 2$. Take any $i_{0} \in A$ and put $A^{\prime}=A \backslash\left\{i_{0}\right\}$. Since $y_{i_{0}}<2\left(q-q^{2}\right)$, then

$$
\sum_{i \in A^{\prime}} y_{i}=\sum_{i \in A} y_{i}-y_{i_{0}}>1-q^{2}-2\left(q-q^{2}\right)=(1-q)^{2} .
$$

Note that $\sum_{i \in A^{\prime}} y_{i} \in F_{k-1}$. If $\sum_{i \in A^{\prime}} y_{i} \neq 1-q$, we have a contradiction. If the equality $\sum_{i \in A^{\prime}} y_{i}=1-q$ holds, we can take another index $i_{1} \neq i_{0}$ instead of $i_{0}$ and we can put $A^{\prime \prime}=A \backslash\left\{i_{1}\right\}$. Then $\sum_{i \in A^{\prime \prime}} y_{i} \in F_{k-1}$ and $\sum_{i \in A^{\prime \prime}} y_{i} \in\left((1-q)^{2}, 1-q\right) \cup\left(1-q, 1-q^{2}\right)$, and we reach a contradiction as well.

Case 2. $m>\frac{1}{q}$ and $\sum_{i=1}^{m} y_{i} \leq 1-q+\left(2 q-2 q^{2}\right)$. Since $y_{1}>q-q^{2}$,

$$
\sum_{i=1}^{m} y_{i}>m\left(q-q^{2}\right)>\frac{1}{q}\left(q-q^{2}\right)=1-q .
$$

Fix $i_{0} \leq m$. If $\sum_{i \neq i_{0}} y_{i}>1-q$ then $\sum_{i \neq i_{0}} y_{i}>1-q^{2}$, because $\left(1-q, 1-q^{2}\right) \cap F_{k}=\emptyset$. Therefore

$$
\sum_{i \leq m} y_{i}=\sum_{i \neq i_{0}} y_{i}+y_{i_{0}}>1-q^{2}+q-q^{2}
$$

which contradicts the assumption $\sum_{i=1}^{m} y_{i} \leq 1-q+\left(2 q-2 q^{2}\right)$. Hence $\sum_{i \neq i_{0}} y_{i} \leq 1-q$. Since $\sum_{i \neq i_{0}} y_{i}>1-q^{2}$ and $\left(1-q, 1-q^{2}\right) \cap F_{k}=\emptyset$ it means that $\sum_{i \neq i_{0}} y_{i}=1-q$.

Thus we have proved that $\sum_{i \neq i_{0}} y_{i}=1-q$ for any $i_{0} \leq m$. It gives a contradiction because $y_{i}$ are different from each other.

Case 3. $m \leq \frac{1}{q}$. We will consider separately $N_{q}^{H}$ for $q>1 / 3$ and for $q \leq 1 / 3$. Suppose that $q>1 / 3$. It means that $m \leq 2$. The assumption that $m=1$ immediately leads to a contradiction. Thus there exist exactly two elements $x_{k-1}, x_{k-2} \in\left(q-q^{2}, 2\left(q-q^{2}\right)\right]$. Note that for $q \in[1 / 3,1]$ the inequality $(1-q)^{2} \leq 2\left(q-q^{2}\right)$ holds. Moreover, $\left((1-q)^{2}, 1-q\right) \cap F_{k-1}=\emptyset$. Therefore there are exactly two elements of the sequence $\left(x_{i}\right)$ in the interval $\left(q-q^{2}, 1-q\right)$. It follows that

$$
(q, 1-q) \subset\left(x_{k-1}+\left(N_{q}^{H} \cap\left[0, x_{k}\right]\right)\right) \cup\left(x_{k-2}+\left(N_{q}^{H} \cap\left[0, x_{k}\right]\right)\right) .
$$

Note that the length of the longest component of $N_{q}^{H} \cap\left[0, x_{k}\right]$ is at most $q^{2}(1-2 q)$. If $q<\sqrt{2}-1$ then $q^{2}<0.42^{2}<1 / 5$ which gives a contradiction with Lemma 6(ii). For $q \geq \sqrt{2}-1$ we use the inequality $q^{2}<1 / 4$ and obtain a contradiction with Lemma 6)(iii).

Suppose now that $q \leq 1 / 3$ and $x_{k}=x_{k-1}$. Then $y_{m}=x_{k}+x_{k-1}=2 q-2 q^{2}$. First assume that $n+2 \notin H$ and put $H^{\prime \prime}=(H-2) \cap \mathbb{N}$. By Lemma 6(i) the longest interval $I$ contained in the translations $\bigcup_{i=1}^{m-1}\left(y_{i}+q^{-n+3} N_{q}^{H^{\prime \prime}}\right)$ has the length smaller than $q^{2}\left((m-1)(1-2 q)+(m-2) q^{2}\right)$. If the left endpoint of $I$ equals $q$, then the right endpoint of $I$ is smaller than

$$
q+q^{2}\left((m-1)(1-2 q)+(m-2) q^{2}\right)=q+q^{2}\left(m(1-2 q)+(m-1) q^{2}\right)-q^{2}(1-q)^{2}
$$




$$
\begin{gathered}
=q+q^{2}\left(m\left(1-2 q+q^{2}\right)-q^{2}\right)+q^{2}(1-q)^{2} \leq q+q\left(1-2 q+q^{2}\right)-q^{4}-q^{2}(1-q)^{2} \\
\leq 2 q-2 q^{2}+q^{3}-q^{2}(1-q)^{2} .
\end{gathered}
$$

On the other hand, the next translate $y_{m}+q^{-n+3} N_{q}^{H^{\prime \prime}}$ cannot cover interval $\left[2 q-2 q^{2}, 2 q-\right.$ $\left.2 q^{2}+q^{3}\right]$. Therefore the union of all translations cannot cover the interval $\left[2 q-2 q^{2}+q^{3}-\right.$ $\left.q^{2}(1-q)^{2}, 2 q-2 q^{2}+q^{3}\right]$, and we reach a contradiction. Note that if $n+2 \in H$, then the estimation is even sharper.

Finally, suppose that $q \leq 1 / 3, m \leq \frac{1}{q}$ and $x_{k}<x_{k-1}$. Then $y_{m}$ is one of $x_{i}$. Assume as before that $n+2 \notin H$. By Lemma 6(i) the longest interval $I$ contained in the translations $\bigcup_{i=1}^{m}\left(y_{i}+q^{-n+3} N_{q}^{H^{\prime \prime}}\right)$ has the length smaller than $q^{2}\left(m(1-2 q)+(m-1) q^{2}\right)$. If the left endpoint of $I$ equals $q$, then the right endpoint of $I$ is smaller than $2 q-2 q^{2}+q^{3}$. Since $x_{k}<x_{k-1}$, then $x_{k}+x_{k-1}>2 q-2 q^{2}$ and the translate $x_{k}+x_{k-1}+q^{-n+3} N_{q}^{H^{\prime \prime}}$ cannot cover the interval $\left[2 q-2 q^{2}+q^{3}, x_{k}+x_{k-1}+q^{3}\right]$. As before, we reach a contradiction.

Corollary 8. No regular Cantorval $N_{q}^{H}$, with $1 \notin H$ and $2 \in H$, is isometric to an achievement set of a sequence.

In fact, any set $E$ isometric to the symmetric set of the form $N_{q}^{H}$ is of the form $E=N_{q}^{H}+t$ for some $t \in \mathbb{R}$. If $E=E\left(x_{n}\right)$ for an absolutely summable sequence $\left(x_{n}\right)$, then $0 \in E$ and hence $t \leq 0$. More precisely, $t$ is the sum of all negative terms of $\left(x_{n}\right)$. Then (see for example [5]) $N_{q}^{H}=E\left(\left|x_{n}\right|\right)$ which gives the contradiction with our theorem.

Remark 9. In the previous theorem it is sufficient to assume that $n-1 \in H, n \notin H$ and $n+1 \in H$ for some $n$ (instead of the assumption $1 \notin H$ and $2 \in H$ ). In fact, let us consider the part $\widetilde{N}=\left[0, q^{n-1}\right] \cap N_{q}^{H}$ of the set $N_{q}^{H}$, with nonremoved middle closed interval $J$ and with removed next intervals $G_{0}$ and $G_{1}$ lying on the left and right of $J$, respectively. To simplify the notation, let us assume (by considering rescaling $q^{-n+1} N_{q}^{H}$ of $N_{q}^{H}$ ) that the right end of $N$ is equal to one. Then $J=[q, 1-q], G_{0}=\left(q^{2}, q-q^{2}\right)$ and $G_{1}=\left(1-q+q^{2}, 1-q^{2}\right)$. Because $n-1 \in H$, one is the left end of the gap which is the longest one from the left. Hence, by the assumption that $N_{q}^{H}=E\left(x_{n}\right)$ and by Lemma 5, we have $1=r_{z}$ for some $z \in \mathbb{N}$, and consequently $\tilde{N}=E\left(x_{n}: n>z\right)$. So we can repeat the conclusion that $F_{k} \cap\left[1-q, 1-q^{2}\right)=$ $\{1-q\}$ and $\left(1-q-\left(q-q^{2}\right), 1-q\right) \cap F_{k-1}=\left((1-q)^{2}, 1-q\right) \cap F_{k-1}=\emptyset$. The rest of the proof runs as before.

It is also worth noting that if $q \leq 1 / 3$ then we do not need any assumptions on $H$. Indeed, since $N_{q}^{H}$ is regular, there exists $n$ such that $n \notin H$ and $n+1 \in H$. By the inequality $q \leq 1 / 3$ and the assumption $N_{q}^{H}=E\left(x_{n}\right)$, we easily conclude that if $q-q^{2}=x_{k}$ then $k=p$ in the notation from the Second Gap Lemma (Lemma 4). Therefore, by this Lemma, we have $1-q^{2} \in F_{k}$ and $1-q+q^{2} \in F_{k}+r_{k}$. Since $\left(1-q+q^{2}\right)-(1-q)=q^{2}=r_{k}$, we have $1-q \in F_{k}$. By $\left(1-q^{2}\right)-(1-q)=q-q^{2}=x_{k}$ we conclude that in fact $1-q \in F_{k-1}$. This way we get once again $F_{k} \cap\left[1-q, 1-q^{2}\right)=\{1-q\}$ and $\left((1-q)^{2}, 1-q\right) \cap F_{k-1}=\emptyset$. 


\section{Attractors of IFS}

Let us recall that all known examples of sequences generating Cantorvals as their achievement sets, are so called multigeometric sequences. We call a sequence multigeometric if it is of the form

$$
\left(k_{1}, k_{2}, \ldots, k_{m}, k_{1} q, k_{2} q, \ldots, k_{m} q, k_{1} q^{2}, \ldots\right)
$$

for some $k_{1}, k_{2}, \ldots, k_{m}, q \in \mathbb{R}$. Following [6], we denote such a sequence by $\left(k_{1}, k_{2}, \ldots, k_{m} ; q\right)$. It was observed in [1], that for the multigeometric sequence $\left(a_{n}\right)=\left(k_{1}, k_{2}, \ldots, k_{m} ; q\right)$ its achievement set $E$ is the unique compact set satisfying the equality $E=\Sigma+q E$ for $\Sigma=\left\{\sum_{n=1}^{m} \varepsilon_{n} k_{n}\right.$ : $\left.\left(\varepsilon_{n}\right) \in\{0,1\}^{m}\right\}$. It is equivalent to the fact that $E$ is an attractor of the IFS consisting of functions $\left\{f_{\sigma}: \sigma \in \Sigma\right\}$ given by formulas $f_{\sigma}(x)=q x+\sigma$. On the other hand, even symmetric set $\Sigma$ does not need to be connected with some multigeometric sequence.

In this section we also need to briefly present the notion of IFS. Let $(X, d)$ be a complete metric space and denote by $K(X)$ space of all nonempty compact subsets of $X$ endowed with the Hausdorff metric $H$ :

$$
H(A, B)=\max \{\sup \{\inf \{d(x, y): y \in A\}: x \in B\}, \sup \{\inf \{d(x, y): y \in B\}: x \in A\}\}
$$

It turns out that $(K(X), H)$ is complete if and only if $X$ is complete.

Definition 10. If $f_{1}, \ldots, f_{n}: X \rightarrow X$ are functions with Lipschitz constants strictly smaller than 1 , then the finite sequence $\mathcal{S}=\left(f_{1}, \ldots, f_{n}\right)$ is called an iterated function system (IFS in short).

If $\mathcal{S}=\left(f_{1}, \ldots, f_{n}\right)$ is an IFS, then by $F_{\mathcal{S}}$ we denote the mapping $F_{\mathcal{S}}: K(X) \rightarrow K(X)$ defined by:

$$
F_{\mathcal{S}}(D)=f_{1}(D) \cup \ldots \cup f_{n}(D) .
$$

It turns out that $F_{\mathcal{S}}$ also has Lipschitz constant smaller than 1 thus, by Banach Fixed Point Theorem, it has the unique fixed point $D_{0}$. We call it an attractor of an IFS $\mathcal{S}$.

This nice idea has been deeply considered in the last 30 years (see, a.e., [11], [10], [4]).

Theorem 11. For any $q \leq 1 / 3$ the set $N_{q}=N_{q}^{2 \mathbb{N}}$ is the attractor of IFS consisting of $4+\left\lceil\frac{1}{q^{2}}\right\rceil$ affine functions given by the formulas

$$
f_{\sigma}(x)=q^{2} x+\sigma
$$

for $\sigma$ 's belonging to some finite set $\Sigma$. (The symbol $\lceil\alpha\rceil$ denotes the smallest integer greater than or equal to $\alpha$.)

Proof. For the sake of clarity let us start with the proof for $q=1 / 3$. Let $\Sigma=\frac{1}{27}\{0,6,8,9,10, \ldots, 16,18,24\}$. It is enough to verify that $N_{1 / 3}=\Sigma+\frac{1}{9} N_{1 / 3}$. Instead of $N_{1 / 3}$ we can consider the set $\tilde{N}=27 N_{1 / 3}$ and $\widetilde{\Sigma}=27 \Sigma$. 

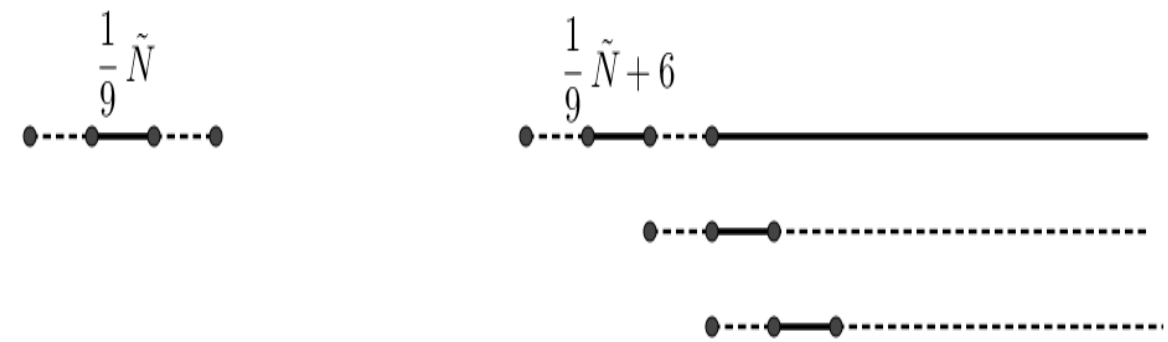

Figure 3

Observe that (see Figure 3):

- $[0,3] \cap \tilde{N}=\frac{1}{9} \tilde{N}$,

- $[6,9] \cap \tilde{N}=\frac{1}{9} \tilde{N}+6$,

- $[8,9] \cap \tilde{N}=\left(\frac{1}{9} \tilde{N}+8\right) \cap[8,9]=\left(\frac{1}{9} \tilde{N}+6\right) \cap[8,9]$,

- The intervals $[9,10],[10,11], \ldots,[17,18]$ are the middle components of $\frac{1}{9} \tilde{N}+8, \frac{1}{9} \tilde{N}+$ $9, \ldots, \frac{1}{9} \tilde{N}+16$ respectively,

- The situation between 18 and 27 is symmetric and analogous to the one between 0 and 9.

Now assume that $q<1 / 3$. Then $N_{q}$ is the union of four translations of the set $q^{2} N_{q}$ and of the middle interval $J$. Denote by $L$ the union of two left copies of $q^{2} N_{q}$, and by $R$ the union of two right ones.

The images of the set $N_{q}$ with respect to the functions

$$
f_{1}(x)=q^{2} x \text { and } f_{2}(x)=q^{2} x+q-q^{2}
$$

cover the set $L$, and the images of the set $N_{q}$ with respect to the functions

$$
f_{3}(x)=q^{2} x+1-q \text { and } f_{4}(x)=q^{2} x+1-q^{2}
$$

cover the set $R$. Now we want to cover the interval $J$ by the images of $J$ with respect to the functions $g_{i}$ given by the formulas $g_{i}(x)=q^{2} x+\sigma_{i}$, for $i=1,2, \ldots, m$.

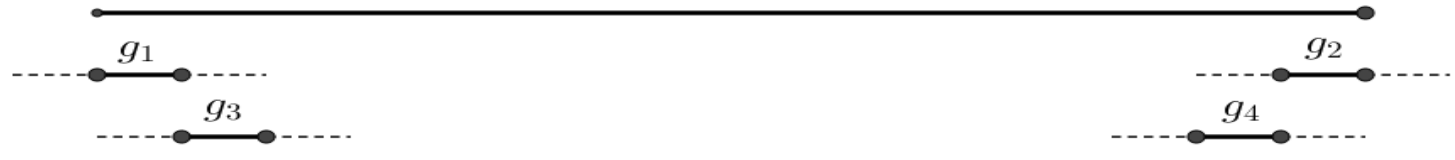

Figure 4

We choose $\sigma_{1}$ such that $g_{1}(q)=q$, and $\sigma_{2}$ such that $g_{2}(1-q)=1-q$. It is worth noting that $g_{1}[L]$ is consistent with $f_{2}[R]$, and $g_{2}[R]$ is consistent with $f_{3}[L]$. The images of $q^{2} N_{q}$ with respect to next functions cover $J$ one by one from the left and from the right. Note that the "middle" two subintervals can be overlapping.

Remark 12. In [1] the authors have analysed the attractor of IFS for the given set $\Sigma$ with respect to the ratio $q$. Making a simple use of their results we easily conclude that for $\Sigma$ described in the proof of Theorem 11 for the Cantorval $N_{1 / 3}$, the attractor $E(\Sigma, q)$ is 
- an interval for $q \geq \frac{1}{5}$,

- a Cantorval for $\frac{1}{9} \leq q<\frac{1}{5}$,

- the set with positive Lebesgue measure for almost all $q \in\left(\frac{1}{13}, \frac{1}{9}\right)$. Unfortunately, we can not say anything on the interior of $E(\Sigma, q)$ in this case,

- measure zero set for some sequence $\left(q_{n}\right)$ decreasing to $\frac{1}{13}$,

- a nowhere dense set for $q \leq \frac{1}{13}$.

For $q>1 / 3$ the situation is more complicated.

Theorem 13. Let $q \in(1 / 3,1 / 2)$. Then the set $N_{q}$ is the attractor of IFS consisting of affine functions with three different ratios.

Proof. We start from the four functions $f_{i}$ exactly as in the proof of the previous theorem. Now we choose a positive integer $n$ satisfying the inequality $q^{2 n}(1-q) \leq 1-2 q$. Then some translation of $q^{2 n}(J \cup R)$ is contained in $J$. We choose $\sigma_{1}$ and $\sigma_{2}$ such that $g_{i}(x)=q^{2 n} x+\sigma_{i}$, $g_{1}[J]$ covers the left and $g_{2}[J]$ covers the right subinterval of $J$. By the self-similarity of the set $N_{q}$ we observe that $g_{1}[L]$ is consistent with $f_{2}[R]$, and $g_{2}[R]$ is consistent with $f_{3}[L]$.

Finally, let $k$ be an integer satisfying the inequalities

- $q^{2 k} \cdot q \leq q^{2 n}(1-2 q)$,

- $q^{2 k}(1-2 q) \leq(1-2 q)-2\left(q^{2 n}(1-2 q)\right)$.

Then we can cover the middle part of $J$ by the images of $J$ with respect to several functions $h_{i}$ of the form $h_{i}(x)=q^{2 k} x+\theta_{i}$, similarly as in the previous proof.

Problem 14. In the last theorem we have used three ratios. It is the natural question if it is possible to use only one ratio. Does there exist a set $N_{q}$ with $q>1 / 3$ which is the attractor of IFS consisting of affine functions with the same ratios?

\section{REFERENCES}

[1] T. Banakh, A. Bartoszewicz, M. Filipczak, E. Szymonik Topological and measure properties of some selfsimilar sets, Topol. Methods Nonlinear Anal., 46 (2015), 1013-1028.

[2] T. Banakh, A. Bartoszewicz, Sz. Głąb, E. Szymonik, Algebraic and topological properties of some sets in $\ell_{1}$, Colloq. Math., 129(1) (2012), 75-85.

[3] M. Banakiewicz, F. Prus-Wiśniowski, M-Cantorvals of Ferens type, to appear in Mathematica Slovaca 67 (2017).

[4] M. F. Barnsley, Fractals everywhere. Academic Press Professional, Boston, MA, 1993.

[5] A. Bartoszewicz, M. Filipczak, F. Prus-Wiśniowski, Topological and algebraic aspects of subsums of series, chapter in Traditional and present-day topics in real analysis, Łódź University Press (2013), 345-366.

[6] A. Bartoszewicz, M. Filipczak, E. Szymonik, Multigeometric sequences and Cantorvals, Cent. Eur. J. Math., 12(7) (2014), 1000-1007.

[7] W. Bielas, S. Plewik, M. Walczyńska, On the center of distances, arXiv:1605.03608v2.

[8] C. Ferens, On the range of purely atomic probability measures, Studia Math., 77(3) (1984), 261-263.

[9] J. A. Guthrie, J. E. Nymann, The topological structure of the set of subsums of an infinite series, Colloq. Math., 55(2) (1988), 323-327.

[10] M. Hata, On the structure of self-similar sets. Japan J. Appl. Math. 2 (1985), 381-414.

[11] J. Hutchinson, Fractals and self-similarity. Indiana Univ. Math. J. 30 (1981), no 5, 713-747. 
[12] R. Jones, Achievement sets of sequences, Amer. Math. Monthly 77(3) (2011), 508-521.

[13] S. Kakeya, On the partial sums of an infinite series, The Science Reports of Tohoku University, 3 (1914) 159-164.

[14] P. Mendes, F. Oliveira, On the topological structure of the arithmetic sum of two Cantor sets, Nonlinearity 7 (2) (1994) 329-343.

[15] Z. Nitecki, The subsum set of a null sequence, arXiv:1106.3779v1.

[16] Z. Nitecki, Cantorvals and subsum sets of null sequences, Amer. Math. Monthly, 122 (2015), no. 9, 862870 .

[17] J. E. Nymann, R. A. Saenz, On a paper of Guthrie and Nymann on subsums on infinite series, Colloq. Math., 83(1) (2000), 1-4.

[18] A. D. Wainshtein, B. Z. Shapiro, Structure of a set of $\bar{\alpha}$-representable numbers, Izv. Vyssh. Uchebn. Zaved. Mat., 5 (1980), 8-11 (in Russian).

E-mail address: artur.bartoszewicz@p.lodz.pl

E-mail address: malfil@math.uni.lodz.pl

E-mail address: szymon.glab@p.lodz.pl

E-mail address: wisniows@univ.szczecin.pl

E-mail address: jswaczyna@wp.pl

Institute of Mathematics, Łódź University of Technology, ul. WólczańskA 215, 93-005 ŁÓDŹ, POLAND

Faculty of Mathematics and Computer Science, Łódź University, ul. Stefana Banacha 22, 90-238 ŁÓDŹ, POLAND

Institute of Mathematics, Łódź University of Technology, Ul. WólczAŃSKA 215, 93-005 ŁóDź, POLAND

Institute of Mathematics, University of Szczecin, Ul. Wielkopolska 15, 70-453 Szczecin, POLAND

Institute of Mathematics, Łódź University of Technology, ul. Wólczańska 215, 93-005 ŁóDŹ, POLAND 